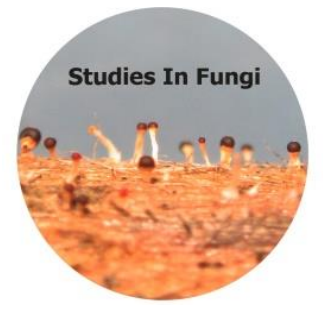

Studies in Fungi 3(1): 126-140 (2018) www.studiesinfungi.org ISSN 2465-4973 Article

Doi 10.5943/sif/3/1/15

Copyright $\odot$ Institute of Animal Science, Chinese Academy of Agricultural Sciences

\title{
Endophytic fungal communities associated with leaves, stems and roots of four medicinal plants in South China
}

\author{
Li T, Deng WQ*, Li TH, Zhang WM, Hosen MI and Song B
}

State Key Laboratory of Applied Microbiology Southern China, Guangdong Provincial Key Laboratory of Microbial Culture Collection and Application, Guangdong Open laboratory of Applied Microbiology, Guangdong Institute of Microbiology, Guangzhou, 510070, China

Li T, Deng WQ, Li TH, Zhang WM, Hosen MI, Song B 2018 - Endophytic fungal communities associated with leaves, stems and roots of four medicinal plants in South China. Studies in Fungi 3(1), 126-140, Doi 10.5943/sif/3/1/15

\begin{abstract}
Amomum villosum, Aquilaria sinensis, Morinda officinalis, and Pogostemon cablin are wellknown medicinal plants in South China for their particular pharmacological activities, but their endophytic fungi have been rarely reported. Here, the endophytic fungal communities associated with the leaves, stems, and roots of the four medicinal plant species were investigated by highthroughput sequencing technology. From the four medicinal plant species, a total of 169,149 sequences (reads) of endophytic fungi were harvested and clustered into 791 operational taxonomic units (OTUs) assigned to the Kingdom Fungi. These OTUs taxonomically spanned five phyla, 27 classes, 75 orders, 167 families, and 291 genera. At the genus level, Phyllosticta, Candida, Zasmidium, and Cryptococcus were the major genera detected in A. villosum; Nigrospora, Tylopilus, Arthrobotrys, Coniosporium, and Corynespora were the dominant genera isolated in A. sinensis; Ochroconis, Cercospora, Aspergillus, and Cyphellophora were the dominant genera detected in M. officinalis; and Cladophialophora, Meira, Sakaguchia, and Penicillium were the major genera isolated in P. cablin. Among the four medicinal plant species, M. officinalis had the highest endophytic fungal diversity $\left(H^{\prime}>4.25\right)$, however $A$. sinensis had the lowest $\left(H^{\prime}<2.37\right)$. There were significant differences in endophytic fungal communities among different organs in the same plant species.
\end{abstract}

Key words - Amomum villosum - diversity - endophytes - Morinda officinalis - traditional Chinese medicine

\section{Introduction}

South China has distinctive ecological and geographic characteristics and abundant medicinal plant resources. Amomum villosum, Aquilaria sinensis, Morinda officinalis, and Pogostemon cablin are well-known medicinal plants owing to their pharmacological activities in Guangdong Province. Amomum villosum (Zingiberaceae) is good for eliminating dampness, strengthening the stomach, and stopping vomiting (Ou 1989). Aquilaria sinensis (Thymelaeaceae) is a source of fragrant wood, and its lignum can form a kind of dark-brown resin under certain pathological condition; this resin is called "Chen Xiang" (a traditional Chinese medicine, TCM) and has been widely used for promoting vital energy circulation, alleviating pain, and relieving dyspnea (Ou 1989). Morinda officinalis (Rubiaceae) has been used for deficiency of kidney-yang manifested as impotence, 
emission, enuresis, frequent micturition, and sterility (Ou 1989). Pogostemon cablin (Labiatae) can be used to eliminate dampness, mitigate summer-heat, stop vomiting, and stimulate the appetite (Ou 1989).

Endophytic fungi comprise an estimate of more than 1 million species based on a ratio of vascular plants to fungal species of 1:4 or 1:5 (Sun et al. 2012); such fungi do not cause apparent negative effects to their hosts and usually can contribute to plant growth and development through producing various secondary metabolites that provide nutrient substances and resist various types of pathogens (Mucciarelli et al. 2003, Ownley et al. 2010). Several bioactive substances are the outcomes of coordinated evolution between plants and endophytic fungi for a long time (Chen et al. 2013). In addition, the endophytes may also produce active substances with potential use to modern medicine (Shekhawat et al. 2013). For example, trichodermic acid, which is an important anticancer drug inducer, can be produced by an endophytic fungus (Trichoderma spirale) isolated from A. sinensis (Li et al. 2012). Crude extractives of zymotic fluid produced by Diaporthe longicolla, an endophytic fungus isolated from $P$. cablin, show activities of resisting the growth of liver, nerve, breast, and lung tumor cells (Wang et al. 2016).

Most previous studies on the diversity of endophytes were conducted with traditional culturedependent methods by means of morphological analysis (Sun et al. 2008, Nalini et al. 2014) and traditional molecular sequencing techniques based on identification of rDNA fragments of sterile strains or isolate communities (Sun et al. 2012, Premalatha \& Kalra 2013). However, numerous non-sporulating and non-culturable fungi, particularly basidiomycetes endophytes, are not easily cultured in artificial media (Sun et al. 2012). Compared with traditional methods, next-generation sequencing techniques, such as 454-pyrosequencing and Illumina sequencing, offer novel and rapid ways for investigating diversity of endophytes directly within the host tissues (Sun et al. 2012, U'Ren et al. 2012), thus allowing the investigation of more endophytic fungus species.

The vast majority of endophytes associated with TCM plants from South China have not been characterized adequately. Based on morphological methods and traditional molecular sequencing techniques, previous studies on endophytes revealed low Shannon indexes $\left(H^{\prime}\right)$, e.g., 269 isolates attributing to 13 taxa were isolated from A. villosum with $H^{\prime}$ of 1.55 (Zhang et al. 2010); 128 isolates vested in 17 genera were isolated from A. sinensis with $H^{\prime}$ of 3.07 (Gong \& Guo 2009); 268 endophytic fungi identified as 12 taxa were isolated from $M$. officinalis, with $H^{\prime}$ of below 2.00 (Zhang et al. 2012); and 1,229 endophytes associated with P. cablin were attributed to 21 genera $\left(H^{\prime}=1.59\right)$ (Zhang 2008). In regard to TCM, few reports relied on high-throughput sequencing, particularly to those from South China. As the first report of endophytes from the TCM plant using this advanced sequencing approach, Sinopodophyllum hexandrum had a high community composition complexity of endophytes (Ning et al. 2016). In addition, abundant effective sequences $(64,179)$ of endophytes in Mentha sp. were also detected successfully (Chen et al. 2016).

High-throughput sequencing can significantly enhance the number, the scope and the depth of endophytic fungal diversity. The aims of the present study were to 1) analyze the endophytic fungal diversity and composition associated with the roots, stems, and leaves of A. villosum, A. sinensis, M. officinalis, and P. cablin from South China; 2) compare the diversity indexes of endophytic fungi using the high-throughput sequencing technology and the traditional methods; 3 ) discuss the differences and similarities in the endophytic fungal diversity and composition among different plant species in the same organ and among different organs in the same plant species.

\section{Materials \& Methods}

\section{Sample collection and surface sterilization}

The four medicinal plants were collected from Guangdong Province (China), which has a tropical and subtropical monsoon climate with the mean annual temperature of $22.6{ }^{\circ} \mathrm{C}$ and the mean annual precipitation of $1845.6 \mathrm{~mm}$ (data in 2015) (Wu et al. 2016). Amomum villosum was collected at the age of 8 months, from Heshui Town, Yangchun City, in April 2015; A. sinensis was collected at the age of 12 months, from Dong Town, Xinyi City, in September 2015; M. officinalis 
at the age of 11 months, was collected from Lubu Town, Gaoyao City, in January 2015; and $P$. cablin at the age of 5 months, was collected from Mashui Town, Yangchun City, in November 2015 (Table 1). The entire bunches of four TCM plants were removed with sterile pruning shears into a sterile plastic bag and transported to the laboratory under refrigeration. Sequencing samples were replicated five times for each organ from the same plant species but different individual. The organs (leaves, stems, and roots) were cut from the samples with a sterile razor blade, and then stored in sterile $10 \mathrm{ml}$ tubes before processing.

Surface sterilization was conducted following the method by Guo et al. (2000). The mud and particles attached to the surface of tissues were washed away with distilled sterile water, and the microbes living on the plant surface were abandoned by immersion in $75 \%$ ethanol for 1 min and $0.1 \% \mathrm{HgCl}_{2}$ for $1 \mathrm{~min}$. The surface-sterilized tissues were washed for 5-7 times with doubledistilled water, and then dried with sterile water absorption pyroxylin. The effect of surface sterilization was tested using the final rinse water spread on potato dextrose agar (PDA).

\section{DNA extraction, PCR amplification, and sample pooling}

DNA samples were extracted from the sterilized tissues following the protocol (Guo et al. 2000). Each sterilized tissue was cut into small pieces. Small pieces of leaves, stems and roots from each plant were considered for DNA extraction using the standard protocol provided by the Power Soil DNA Isolation Kit (Mo Bio Laboratories, Inc). The obtained DNA products were stored at $-80{ }^{\circ} \mathrm{C}$ for PCR amplification.

To amplify the fungal internal transcribed spacer (ITS1) of nuclear ribosomal DNA sequences, a pair of primers ITS1 (5'-TCCGTAGGTGAACCTGCGG-3') and ITS2 (5'-GCTGC GTTCTTCATCGATGC-3') were chosen (White et al. 1990, Gardes \& Bruns 1993). Each PCR reaction contained $10 \mathrm{ng}$ of template DNA, $10 \mu \mathrm{l}$ of $2 \times$ EasyTaq PCR SuperMix (Transgen Bio, Inc), and $1 \mu \mathrm{l}$ of each primer, and then $\mathrm{ddH}_{2} \mathrm{O}$ was added to reach $25 \mu \mathrm{l}$. The PCR cycling conditions were denaturation at $94{ }^{\circ} \mathrm{C}$ for $5 \mathrm{~min}$, followed by 35 cycles of denaturation at $94{ }^{\circ} \mathrm{C}$ for $30 \mathrm{~s}$, annealing at $55{ }^{\circ} \mathrm{C}$ for $30 \mathrm{~s}$, extension at $72{ }^{\circ} \mathrm{C}$ for $1 \mathrm{~min}$, and a final extension at $72{ }^{\circ} \mathrm{C}$ for 8 min. The PCR products were isolated on a $2 \%$ low-melt agarose gel and purified with a MiniBEST Agarose Gel DNA Extraction Kit (Takara Inc.).

The amplicon libraries were sent to Biomarker Tech Co., Ltd., Beijing, China and Genepioneer Biotech Co., Ltd., Nanjing, China for DNA sequencing on an Illumina MiSeq PE250 platform. The sequencing data were deposited in the NCBI Sequence Read Archive (SRP074960).

\section{Bioinformatic read processing and taxonomic classification}

The raw sequence data were analyzed by FLASH (v. 1.2.7, http://ccb.jhu.edu/software/ FLASH/), which is a fast and accurate program, to increase the length of reads by overlapping and merging paired reads from fragments shorter than twice the length of reads. The following programs were also used for the analysis: Trimmomatic (v. 0.36, http://www.usadellab.org/cms/? page $=$ trimmomatic), which is a more flexible and efficient pre-processing tool to correctly handle paired-end data, UCHIME (v. 4.2, http://drive5.com/uchine), which is an algorithm for detecting and removing chimeric sequences, and QIIME (v. 1.9.1, https://pypi.python.org/pypi/ qiime), which is a software for demultiplexing, quality filtering, OTU picking, taxonomic assignment, phylogenetic reconstruction, diversity analyses and visualizations. The sequence data were spliced and formed as the raw reads. The raw reads of the plant species and putative chimeric sequences were removed, and the effective reads were accepted with an average quality score above 20 and length longer than $100 \mathrm{bp}$. Then, the effective reads were clustered on the basis of a 97\% similarity threshold to build operational taxonomic units (OTUs). The resulting file was parsed to separate the data for each sample. OTUs were classified by taxonomic affiliation with "Classify. Seqs" using the data base UNITE (http://unite.ut.ee/repository.phh) with a bootstrap confidence cutoff value $70 \%$. The following criteria were used to implement the OTUs: bootstrap scores below the $70 \%$ were left as "unassigned", scores between $95 \%$ and $70 \%$ 
were labeled as the family, order, class and higher classification levels, scores between $97 \%$ and 95\% were identified as the genus level, and scores above $97 \%$ were accepted as the species and genus levels.

\section{Statistical analyses of fungal community composition}

To calculate the alpha-diversity, the effective OTUs were analyzed by two indices of the OTUs richness (i.e., Chao1 \& Shannon indices) using Mothur (v. 1.30, http://www.mothur.org/). To determine the relationships among the endophytes in the four medicinal plant species, Venn diagrams (http://bioinformatics.psb.ugent.be/webtools/Venn/) and non-metric multi-dimensional scaling (NMDS) (v. 7, PC-ORD, http://www.pcord.com/) were used to analyze the differences and similarities of endophytic fungal OTUs. To estimate the similarities among fungal communities, Sorenson's pairwise similarity coefficient $(C S)$ was also followed: $C S(\%)=$ $2 j /(a+b) \times \%$, where $j$ is the OTU numbers shared by two different species, and $a$ and $b$ represent the OTU numbers of sample a and sample $b$, respectively.

\section{Results}

\section{Taxonomic analyses of endophytic fungi}

The raw sequence data of the 12 samples consisted of 1,381 OTUs of 394,791 reads. After removing chimeric sequences and reads assigned to nontarget organisms, 169,149 reads clustered into 791 OTUs remained, which were assigned to the Kingdom Fungi.

According to the data based on GenBank and Index Fungorum, the OTUs were classified at different taxonomic levels. The 791 OTUs could be assigned to phylum level for 779 OTUs, to class level for 732 OTUs, to order level for 705 OTUs, to family level for 667 OTUs, to genus level for 691 OTUs, and to unknown taxa for 12 OTUs (241 reads). These OTUs taxonomically spanned five phyla, 27 classes, 72 orders, 167 families, and 291 genera (Table S).

The most heavily-sequenced phylum associated with leaves, stems, and roots from all four plant species was Ascomycota (116,381 reads, 404 OTUs). Members of Basidiomycota were in second place (50,956 reads, 330 OTUs). Zygomycota (1,321 reads, 30 OTUs), Glomeromycota (205 reads, 9 OTUs) and Chytridiomycota (45 reads, 6 OTUs) were the minor phyla in term of number of reads and OTUs (Fig. 1, Table S).

At the class level, Dothideomycetes (14,915 reads, 127 OTUs), Eurotiomycetes (26,740 reads, 117 OTUs), and Sordariomycetes (54,679 reads, 74 OTUs) were the most abundant and diverse within Ascomycota. Agaricomycetes (34,436 reads, 195 OTUs) and Tremellomycetes (2,443 reads, 43 OTUs) were the most abundant and diverse within Basidiomycota. In Zygomycota, Mucoromycetes (233 reads, 21 OTUs) had the richest OTUs, whereas Mortierellomycetes (1096 reads, 8 OTUs) had the richest reads (Table 2).

More than $1 \%$ (>7 OTUs) of the total number of OTUs were revealed as dominant fungi in terms of fungal orders (Fig. 1): Pleosporales (4,719 reads, 80 OTUs), Capnodiales (1,409 reads, 16 OTUs), Mycosphaerellales (2,352 reads, 8 OTUs) and Venturiales (3,722 reads, 8 OTUs) within Dothideomycetes; Eurotiales (4,349 reads, 65 OTUs) and Chaetothyriales (21,769 reads, 40 OTUs) within Eurotiomycetes; Helotiales (350 reads, 14 OTUs) within Leotiomycetes; Saccharomycetales (1,223 reads, 23 OTUs) within Saccharomycetes; Hypocreales (964 reads, 25 OTUs) and Sordariales (346 reads, 22 OTUs) within Sordariomycetes; Agaricales (10,584 reads, 81 OTUs), Polyporales (825 reads, 35 OTUs), Boletales (19,768 reads, 20 OTUs), Auriculariales (619 reads, 16 OTUs), Cantharellales (186 reads, 11 OTUs) and Russulales (310 reads, 10 OTUs) within Agaricomycetes; Malasseziales (365 reads, 15 OTUs) within Malasseziomycetes; Tremellales (2,336 reads, 40 OTUs) within Tremellomycetes; Mucorales (168 reads, 17 OTUs) and Mortierellales (1,096 reads, 8 OTUs) within Mortierellomycetes and Mucoromycetes, respectively.

The commonly detected fungal genera (>1\% dominant fungi and $>1,000$ reads) were Nigrospora (52,416 reads, 2 OTUs), Tylopilus (19,449 reads, 3 OTUs), Arthrobotrys (16,847 
reads, 4 OTUs), Cladophialophora (14,261 reads, 8 OTUs), an unknown genus of Agaricales (9,021 reads, 1 OTU), Meira (7,051reads, 4 OTUs), Sakaguchia (4,735 reads, 4 OTUs), Coniosporium (4,213 reads, 4 OTUs), Ochroconis (3,722 reads, 8 OTUs), Corynespora (2,525 reads, 1 OTU), Cercospora (2,327 reads, 4 OTUs), Phyllosticta (2,203 reads, 1 OTU), Aspergillus (2,084 reads, 18 OTUs), Penicillium (1,836 reads, 28 OTUs), Cyphellophora (1,635 reads, 2 OTUs), and an unknown genus of Tremellales (1,197 reads, 1 OTU) (Table S).

\section{Diversity and similarity of endophytic fungi}

Chaol and Shannon were used to evaluate and compare the diversity of fungal community richness among the 12 plant samples. Chao1 index ranged from 43.77 to 256.45 , and Shannon's index ranged from 2.22 to 4.69 (Table 3 ), thus indicating that the diversity vary among the 12 samples. All the four plant species were found to harbor 20 different endophyte orders, such as Capnodiales, Pleosporales, Helotiales, Agaricales, and Polyporales.

Among the 791 OTUs in this study, 617 were detected from only one plant species, 142 from two plant species, 23 from three plant species, and 9 from all four plans, which indicated that a number of distinctive OTUs that belonged to only one plant species (Fig. 2a). Sorenson's similarity coefficients for endophytic fungal community composition were low among the four plant species (Table 4). The highest similarity (39.42\%) was between A. sinensis and P. cablin, and the lowest similarity (8.27\%) was between A. villosum and M. officinalis (Table 4). These low Sorenson's similarity coefficients indicated that the fungi have different distributions among the plant species.

The OTU number per sample varied among organs of four plant species, ranging from 41 to 213 (Table 3). In all samples, the leaves of $A$. villosum hosted the least OTUs number, whereas the roots of $P$. cablin hosted the most. In A. villosum, 108 OTUs were distributed in the different organs; roots hosted the most OTUs (90 OTUs), followed by stems (46 OTUs) and leaves (41 OTUs) (Fig. 3a); the three different organs shared 23.14\% (25 OTUs) of the total OTUs which taxonomically spanned three phyla, 17 classes, 36 orders, 64 families, and 75 genera; the dominant genera were Phyllosticta, Candida, Zasmidium, and Cryptococcus. In A. sinensis, leaves hosted the most OTUs (162 OTUs), followed by stems (147 OTUs) and roots (112 OTUs) (Fig. $3 b$ ), but only $14.13 \%$ (39 OTUs) of total OTUs numbers overlap in its three organs; the OTUs taxonomically spanned five phyla, 24 classes, 54 orders, 104 families, and 149 genera; the dominant genera were Nigrospora, Tylopilus, Arthrobotrys, Coniosporium, and Corynespora. In M. officinalis, roots hosted the most OTUs (199), followed by leaves (133) and stems (121) (Fig. 3c); three different organs shared $21.51 \%$ (60 OTUs) of the total OTUs; the total OTUs taxonomically spanned five phyla, 22 classes, 49 orders, 103 families, and 154 genera; the dominant genera were Ochroconis, Cercospora, Aspergillus, and Cyphellophora; therein Ochroconis was the only isolate in the roots with the most reads (3666). In P. cablin, roots hosted most OTUs (231), followed by stems (200) and leaves (173) (Fig. 3d), and three organs shared $22.45 \%$ of the total OTUs; the total OTUs vested in $P$. cablin taxonomically spanned five phyla, 21 classes, 58 orders, 116 families, and 168 genera; as the dominant genera, Cladophialophora, Meira, Sakaguchia, Penicillium, and the unclassified genera of Agaricales were isolated from the P. cablin (Table S).

Based on Jaccard dissimilarities calculated from the read numbers of the 791 inferred OTUs among samples of communities from four plants, the NMDS analyses indicated that the fungal communities were more or less host-specific and exhibited a trend that the endophyte diversity of A. sinensis was similar to that of P. cablin. They were both in the third quadrant (Fig. 4). The data of NMDS and Sorenson's similarity coefficients showed the similar results (Table 4). The endophyte genera of $A$. sinensis and $P$. cablin including leaves, stems and roots shared more OTUs (122 OTUs) (Fig. 2a), therein, stems shared most 55 OTUs, leaves shared 33 and roots shared 30 (Fig. 2b). However, the endophytic fungal OTUs in A. villosum differed from those of $M$. officinalis, only 16 OTUs were shared by both plants, even 1 OTU (an unknown genus of Pleosporales) was shared by their leaves (Fig. 2a, Table S). 


\section{Discussion}

\section{High-throughput sequencing significantly enhances the characterization of fungal diversity}

The present study revealed the rich bio-diversity and distinction of endophytes within the four TCM plant species in South China. The high diversity $\left(H^{\prime}=2.22-4.69\right)$ showed that complex fungal networks exist in their tissues. The 169,149 effective sequences vested into 791 OTUs, spanning five phyla, 27 classes, 75 orders, 167 families, and 291 genera. By contrast, the known diversity of endophytes within these four TCM plant species (A. villosum, A. sinensis, $M$. officinalis and $P$. cablin) was much lower $\left(H^{\prime}=1.55-3.07\right)$ when the traditional methods were used (Zhang 2008, Gong \& Guo 2009, Zhang et al. 2010, Zhang et al. 2012). Likewise, Sun et al. (2008) reported that the known diversity of endophytes inhabiting six rare and important TCM plants (Eucommia ulmoides, three species of Forsythia, Berberis poiretii, and Rhus potaninii) growing in Northern China was also lower $\left(H^{\prime}=0.98-1.60\right)$. However, even in extreme environmental conditions, such as in the High Arctic Zone, highly diverse endophyte communities $\left(H^{\prime}=2.24-4.24\right)$ existing in the Arctic plants could be detected when using the high-throughput sequencing approach (Zhang \& Yao 2015).

Several common fungi, such as Colletotrichum, Phyllosticta, Phomopsis, Chaetomium, Alternaria, Fusarium, and Hyphomycete, were isolated from A. villosum by the traditional technique, and Colletotrichum had the highest frequency (Zhang et al. 2010). When highthroughput sequencing was used, Phyllosticta had the most reads in A. villosum and became the most common genus, with 914 reads in leaves, 696 in stems, and 593 in roots. In the whole plant of A. villosum, 75 genera with 6,422 reads and 108 OTUs were investigated, including Phyllosticta, Candida, Malassezia, Zasmidium, Cryptococcus, Sigarispora, Penicillium, Boletus, and Acremonium, which showed a much richer, more comprehensive and detailed fungal community diversity.

Fusarium, Glomerularia, Cladosporium, Cephalosporium, Botryosphaeria, and Colletotrichum were the dominant endophytic fungi isolated from A. sinensis when using the traditional technique (Wang et al. 2005, Gong \& Guo 2009, Wang et al. 2009); therein, Colletotrichum spp. and Fusarium spp. were relevant to the agalloch part (stems and roots). This survey showed that Fusarium only inhabited the stems and Colletotrichum only inhabited the roots respectively, with 1 read and 24 reads. Taxa such as Alternaria, Fusarium, Trichoderma, and other commonly found genera were abundant in culture-based studies, but only a few reads or no recovered data were found with the culture-independent methods. An endophytic fungus of Trichoderma, isolated from A. sinensis, only exist in its leaves with 78 reads. These species were fast-growing fungi. Thus, they were recovered abundantly and isolated preferentially on PDA media, although they were originally in small amounts; by contrast, the unculturable and slow growing fungi were not detected in traditional endophytes isolation studies (Duong et al. 2006, Mohamed et al. 2010). Fungal communities in agarwood differed from those in the tissues that did not become agarwood or could not produce fragrance. The genetic material age of the tested A. sinensis was only four months, which was too young to produce fragrant ingredients. Actually, traditional approach could have missed many members of the fungal communities; as a result, merely 17 genera of fungi colonizing in A. sinensis had been reported (Gong \& Guo 2009). By contrast, 149 genera of fungi were revealed through next-generation sequencing in the current study.

The roots, which host the distinctive endophytic fungi and most OTUs, were the effective medicinal parts of $M$. officinalis; and the relationships between the fungal taxa and the medicinal efficacy (treating rheumatoid arthritis and impotence) need further investigation. According to previous reports from Dreyfuss \& Chapela (1994), Panda et al. (2016), Aspergillus and Penicillium, so called "creative fungi", could produce various secondary metabolites, which mainly existed in the whole plant of M. officinalis in the present study. Metschnikowia, a kind of yeast only isolated from the roots of $M$. officinalis, could resist certain postharvest pathogens of 
apple fruits, such as Botrytis sp., Penicillium sp., Alternaria sp., and Monilia sp. (Spadaro et al. 2004).

Similarly, the leaves and stems were the medicinal parts of $P$. cablin, with peculiar fragrance, for treating summer heat. The leaves and stems hosted smaller number of OTUs than the roots. Cladophialophora was the dominant genus in the leaves, stems, and roots of $P$. cablin. Traditionally isolated from $P$. cablin, Diaporthe spp. show anti-tumor activities (Badali et al. 2008, Sun et al. 2012) and only existed in the roots with 40 reads in the present study.

\section{The specificity and universality of endophyte communities}

Previous studies reported that the composition of fungal communities associated with above-ground plant tissues depends on the host species (Sun et al. 2012, Higgins et al. 2007). In the present study, the NMDS and Venn diagrams analyses of OTUs revealed a specific relevance exists between the host species and their endophytes. The differentiation of fungal communities possibly depends on the different types of plant species and their metabolic substances and substrates (Zhang et al. 2011). A. sinensis and P. cablin can produce some similar major chemical compounds, such as sterols, terpenes, flavonoids, benzophenones, but less or even no polysaccharides or carbohydrates ( $\mathrm{Li}$ et al. 2011, Chen et al. 2012); on the account of the similar characteristics in metabolic substances and substrates, very similar diversity and communities of endophytes were noticed in these two medicinal plants; the common genera in both plant species are Candida, Mortierella, Arthroderma, Microdochium, etc. However, M. officinalis has unique compound components, its major chemical constituents are carbohydrate, anthraquinones, iridoid glycosides, organic acids, trace element, etc. (Lin et al. 2010); meanwhile, endophytes in M. officinalis have more distinctive OTUs (224) than those of other three species (Fig. 2a). Essential oils, as a kind of volatile chemical compounds, were both extracted from A. villosum and $P$. cablin, though with different chemical structures or ingredients (Fu et al. 2011, $\mathrm{Li}$ et al. 2011); around 30 endophytic fungal OTUs were shared in A. villosum and P. cablin (Fig. 2a), which represented with the common genera Cryptococcus, Penicillium, Pluteus, etc. In the present study, the similarities or differences of endophyte communities in plant species, perhaps due to the similar or different metabolic substances and substrates produced by their host TCM plants. Interestingly, the endophytes among different organs in A. sinensis (ligneous plant) shared a much lower percentage of OTUs than those of other plant species (herbaceous plant) which have very similar percentage of OTUs.

In summary, the high-throughput sequencing offered convenient and fast way for elucidating fungal community diversity. Our results illustrated that the four indigenous TCM plant species (A. villosum, A. sinensis, M. officinalis, and $P$. cablin) and their respective organs (leaves, stems, and roots) harbor more abundant endophytic fungal species compared to traditional methods. Endophytic fungal diversity and communities have significant different among the four plant species. Meanwhile, endophytes among different organs in the same host plant species were significant different. Considering that only four TCM plant species were collected in the present study, further endophyte studies should include more plant species. In view the medicinal efficacies and metabolites of the TCM plants, our results may be beneficial to the future researches on the TCM plants in field of medicament and human health.

Table 1 Samples information of the four tradition Chinese medicine (TCM) plants

\begin{tabular}{lllccc}
\hline Plant species & Organs & Coordinations & $\begin{array}{c}\text { Height } \\
(\mathbf{m})\end{array}$ & $\begin{array}{c}\text { Temperature } \\
\left({ }^{\circ} \mathbf{C}\right)\end{array}$ & $\begin{array}{c}\text { Relative } \\
\text { humidity }\end{array}$ \\
\hline $\begin{array}{l}\text { Amomum } \\
\text { villosum }\end{array}$ & Roots & $\begin{array}{l}22^{\circ} 06^{\prime} 34.02^{\prime \prime} \mathrm{N} ; \\
111^{\circ} 42^{\prime} 20.64 ” \mathrm{E}\end{array}$ & 8 & 21 & $52 \%$ \\
A. villosum & Stems & $\begin{array}{l}22^{\circ} 06^{\prime} 34.02^{\prime \prime} \mathrm{N} ; \\
111^{\circ} 42^{\prime} 20.64 ” \mathrm{E}\end{array}$ & 8 & 21 & $52 \%$ \\
A. villosum & Leaves & $\begin{array}{l}22^{\circ} 06^{\prime} 34.02^{\prime \prime} \mathrm{N} ; \\
111^{\circ} 42^{\prime} 20.64 ” \mathrm{E}\end{array}$ & 8 & 21 & $52 \%$
\end{tabular}


Table 1 Continued.

\begin{tabular}{|c|c|c|c|c|c|}
\hline Plant species & Organs & Coordinations & $\begin{array}{c}\text { Height } \\
(\mathrm{m})\end{array}$ & $\begin{array}{c}\text { Temperature } \\
\left({ }^{\circ} \mathbf{C}\right)\end{array}$ & $\begin{array}{r}\begin{array}{r}\text { Relative } \\
\text { humidity }\end{array} \\
\end{array}$ \\
\hline $\begin{array}{l}\text { Aquilaria } \\
\text { sinensis }\end{array}$ & Roots & $\begin{array}{l}22^{\circ} 21^{\prime} 12.50^{\prime \prime} \mathrm{N} \\
110^{\circ} 56^{\prime} 29.21^{\prime \prime} \mathrm{E}\end{array}$ & 85 & 26 & $62 \%$ \\
\hline A. sinensis & Stems & $\begin{array}{l}22^{\circ} 21^{\prime} 12.50^{\prime \prime} \mathrm{N} \\
110^{\circ} 56^{\prime} 29.21^{\prime \prime} \mathrm{E}\end{array}$ & 85 & 26 & $62 \%$ \\
\hline A. sinensis & Leaves & $\begin{array}{l}22^{\circ} 21^{\prime} 12.50^{\prime \prime} \mathrm{N} \\
110^{\circ} 56^{\prime} 29.21^{\prime \prime} \mathrm{E}\end{array}$ & 85 & 26 & $62 \%$ \\
\hline $\begin{array}{l}\text { Morinda } \\
\text { officinalis }\end{array}$ & Roots & $\begin{array}{l}23^{\circ} 10^{\prime} 40.71 ” \mathrm{~N} \\
112^{\circ} 17^{\prime} 39.83^{\prime \prime} \mathrm{E}\end{array}$ & 77 & 17 & $65 \%$ \\
\hline M. officinalis & Stems & $\begin{array}{l}23^{\circ} 10^{\prime} 40.71 ” \mathrm{~N} \\
112^{\circ} 17^{\prime} 39.83^{\prime \prime} \mathrm{E}\end{array}$ & 77 & 17 & $65 \%$ \\
\hline M. officinalis & Leaves & $\begin{array}{l}23^{\circ} 10^{\prime} 40.71 ” \mathrm{~N} \\
112^{\circ} 17^{\prime} 39.83^{\prime \prime} \mathrm{E}\end{array}$ & 77 & 17 & $65 \%$ \\
\hline $\begin{array}{l}\text { Pogostemon } \\
\text { cablin }\end{array}$ & Roots & $\begin{array}{l}22^{\circ} 18^{\prime} 17.90 ” \mathrm{~N} \\
111^{\circ} 52^{\prime} 21.444^{\prime \prime} \mathrm{E}\end{array}$ & 54 & 19 & $60 \%$ \\
\hline P. cablin & Stems & $\begin{array}{l}22^{\circ} 18^{\prime} 17.90 ” \mathrm{~N} \\
111^{\circ} 52^{\prime} 21.44^{\prime \prime} \mathrm{E}\end{array}$ & 54 & 19 & $60 \%$ \\
\hline P. cablin & Leaves & $\begin{array}{l}22^{\circ} 18^{\prime} 17.90 ” \mathrm{~N} \\
111^{\circ} 52^{\prime} 21.44^{\prime \prime} \mathrm{E}\end{array}$ & 54 & 19 & $60 \%$ \\
\hline
\end{tabular}

Table 2 Overview of the taxonomic composition of endophytic fungal communities at the Class level found in four tradition Chinese medicine (TCM) plant species

\begin{tabular}{lllllll}
\hline & $\begin{array}{l}\text { reads } \\
(\%)\end{array}$ & $\begin{array}{l}\text { OTUs } \\
(\%)\end{array}$ & $\begin{array}{l}\text { Amomum } \\
\text { villosum }(\%)\end{array}$ & $\begin{array}{l}\text { Aquilaria } \\
\text { sinensis } \\
(\%)\end{array}$ & $\begin{array}{l}\text { Morinda } \\
\text { officinalis } \\
(\%)\end{array}$ & $\begin{array}{l}\text { Pogostemon } \\
\text { cablin }(\%)\end{array}$ \\
\hline Ascomycota & $\mathbf{6 8 . 8}$ & $\mathbf{5 1 . 1}$ & $\mathbf{7 9 . 9 9}$ & $\mathbf{7 7 . 5}$ & $\mathbf{7 4 . 6 1}$ & $\mathbf{4 4 . 8 8}$ \\
Dothideomycetes & 8.818 & 16.056 & 58.004 & 3.456 & 40.035 & 2.202 \\
Eurotiomycetes & 15.809 & 14.791 & 3.504 & 5.277 & 23.362 & 39.069 \\
Lecanoromycetes & 0.017 & 0.632 & - & 0.01 & 0.108 & - \\
Leotiomycetes & 0.444 & 3.161 & 0.903 & 0.355 & 1.471 & 0.193 \\
Orbiliomycetes & 10.075 & 0.632 & - & 16.528 & 1.178 & 0.007 \\
Pezizomycetes & 0.034 & 0.379 & $\mathbf{-}$ & 0.005 & 0.317 & - \\
Saccharomycetes & 0.723 & 2.908 & 15.494 & 0.013 & 0.903 & 0.145 \\
Schizosaccharomycetes & 0.003 & 0.126 & $\mathbf{-}$ & 0.005 & - & - \\
Sordariomycetes & 32.326 & 9.355 & 1.962 & 51.698 & 6.785 & 1.676 \\
Taphrinomycetes & 0.416 & 0.126 & $\mathbf{-}$ & 0.093 & - & 1.379 \\
Unknown Ascomycota & 0.14 & 2.908 & 0.125 & 0.061 & 0.454 & 0.206 \\
Basidiomycota & $\mathbf{3 0 . 1}$ & $\mathbf{4 1 . 7}$ & $\mathbf{1 9 . 8 4}$ & $\mathbf{2 1 . 4 4}$ & $\mathbf{2 3 . 4 9}$ & $\mathbf{5 4 . 2 2}$ \\
Agaricomycetes & 20.358 & 24.652 & 3.924 & 20.46 & 12.661 & 25.436 \\
Agaricostilbomycetes & 0.012 & 0.759 & $\mathbf{-}$ & 0.004 & 0.054 & 0.018 \\
Cystobasidiomycetes & 2.861 & 1.77 & 0.841 & 0.063 & 0.12 & 10.659 \\
Dacrymycetes & 0.002 & 0.126 & $\mathbf{-}$ & - & 0.018 & - \\
Exobasidiomycetes & 4.247 & 1.896 & 0.187 & 0.006 & 0.251 & 16.153 \\
Geminibasidiomycetes & 0.007 & 0.126 & 0.062 & 0.003 & 0.006 & 0.007 \\
Malasseziomycetes & 0.216 & 1.896 & 3.815 & 0.058 & 0.215 & 0.057 \\
Microbotryomycetes & 0.125 & 1.264 & 0.358 & 0.025 & 0.179 & 0.302 \\
Moniliellomycetes & 0.002 & 0.126 & 0.047 & - & - & - \\
Pucciniomycetes & 0.001 & 0.126 & $\mathbf{-}$ & - & 0.012 & - \\
Tremellomycetes & 1.444 & 5.436 & 10.48 & 0.069 & 8.722 & 0.546 \\
\hline
\end{tabular}


Table 2 Continued.

\begin{tabular}{lllllll}
\hline & $\begin{array}{l}\text { reads } \\
(\%)\end{array}$ & $\begin{array}{l}\text { OTUs } \\
(\%)\end{array}$ & $\begin{array}{l}\text { Amomum } \\
\text { villosum }(\%)\end{array}$ & $\begin{array}{l}\text { Aquilaria } \\
\text { sinensis } \\
(\%)\end{array}$ & $\begin{array}{l}\text { Morinda } \\
\text { officinalis } \\
(\%)\end{array}$ & $\begin{array}{l}\text { Pogostemon } \\
\text { cablin }(\%)\end{array}$ \\
\hline Ustilaginomycetes & 0.006 & 0.506 & 0.031 & 0.004 & - & 0.009 \\
Wallemiomycetes & 0.003 & 0.126 & 0.078 & - & - & - \\
Unknown & 0.841 & 2.908 & 0.016 & 0.744 & 1.249 & 1.032 \\
Basidiomycota & & & & & & \\
Chytridiomycota & $\mathbf{0 . 0 3}$ & $\mathbf{0 . 7 6}$ & $\mathbf{0}$ & $\mathbf{0 . 0 1 5}$ & $\mathbf{0 . 0 3}$ & $\mathbf{0 . 0 5 7}$ \\
Blastocladiomycetes & 0.017 & 0.379 & - & 0.006 & - & 0.052 \\
Chytridiomycetes & 0.009 & 0.379 & - & 0.009 & 0.03 & 0.005 \\
Glomeromycota & $\mathbf{0 . 1 2}$ & $\mathbf{1 . 1 4}$ & - & $\mathbf{0 . 0 8}$ & $\mathbf{0 . 0 5}$ & $\mathbf{0 . 2 7}$ \\
Glomeromycetes & 0.121 & 1.138 & - & 0.077 & 0.054 & 0.268 \\
Zygomycota & $\mathbf{0 . 7 8}$ & $\mathbf{3 . 7 9}$ & $\mathbf{0 . 1 4}$ & $\mathbf{0 . 9 5}$ & $\mathbf{0 . 5 5}$ & $\mathbf{0 . 5 7}$ \\
Mortierellomycetes & 0.648 & 1.011 & 0.016 & 0.877 & 0.197 & 0.381 \\
Mucoromycetes & 0.132 & 2.655 & 0.125 & 0.075 & 0.341 & 0.186 \\
Unknown Zygomycota & 0.001 & 0.126 & $\mathbf{-}$ & $\mathbf{-}$ & 0.012 & - \\
Unknown fungi & $\mathbf{0 . 0 0 1}$ & $\mathbf{1 . 5 1 7}$ & $\mathbf{0 . 0 1}$ & $\mathbf{0 . 1 3}$ & $\mathbf{1 . 2 7}$ & $\mathbf{0 . 0 4}$ \\
\hline
\end{tabular}

The second and third columns indicated the percentages of total OTUs and the total number of reads across the four plant species, respectively. The last four columns provide the fungal communities found in the four plant species, which are presented as the percentages of sequence reads

Table 3 Diversity indexes of four plant species of tradition Chinese medicine (TCM) and their respective organs

\begin{tabular}{lllll}
\hline Plant species & Organs & OTU $^{\text {a }}$ & Chao1 $^{\mathbf{b}}$ & $\boldsymbol{H}^{\mathbf{C}}$ \\
\hline Amomum villosum & Roots & 90 & 112.89 & 3.80 \\
A. villosum & Stems & 46 & 54.75 & 2.78 \\
A. villosum & Leaves & 41 & 43.77 & 2.69 \\
Aquilaria sinensis & Roots & 112 & 122.54 & 2.37 \\
A. sinensis & Stems & 147 & 157.36 & 2.32 \\
A. sinensis & Leaves & 162 & 180.59 & 2.22 \\
Morinda officinalis & Roots & 199 & 206.50 & 4.25 \\
M. officinalis & Stems & 121 & 124.00 & 4.48 \\
M. officinalis & Leaves & 133 & 142.85 & 4.69 \\
Pogostemon cablin & Roots & 213 & 239.50 & 3.34 \\
P. cablin & Stems & 200 & 256.45 & 3.37 \\
$P$. cablin & Leaves & 173 & 200.82 & 3.30 \\
\hline . &
\end{tabular}

a. Number of OTUs; b. Chao index; C. Shannon index

Table 4 Sorenson's similarity coefficients for endophytic fungal community composition among the four plant species of tradition Chinese medicine (TCM).

\begin{tabular}{llll}
\hline Plant species & Amomum villosum & Aquilaria sinensis & Morinda officinalis \\
\hline Amomum villosum & - & - & - \\
Aquilaria sinensis & $14.58 \%$ & - & - \\
Morinda officinalis & $8.27 \%$ & $11.53 \%$ & - \\
Pogostemon cablin & $13.30 \%$ & $39.42 \%$ & $11.90 \%$ \\
\hline
\end{tabular}



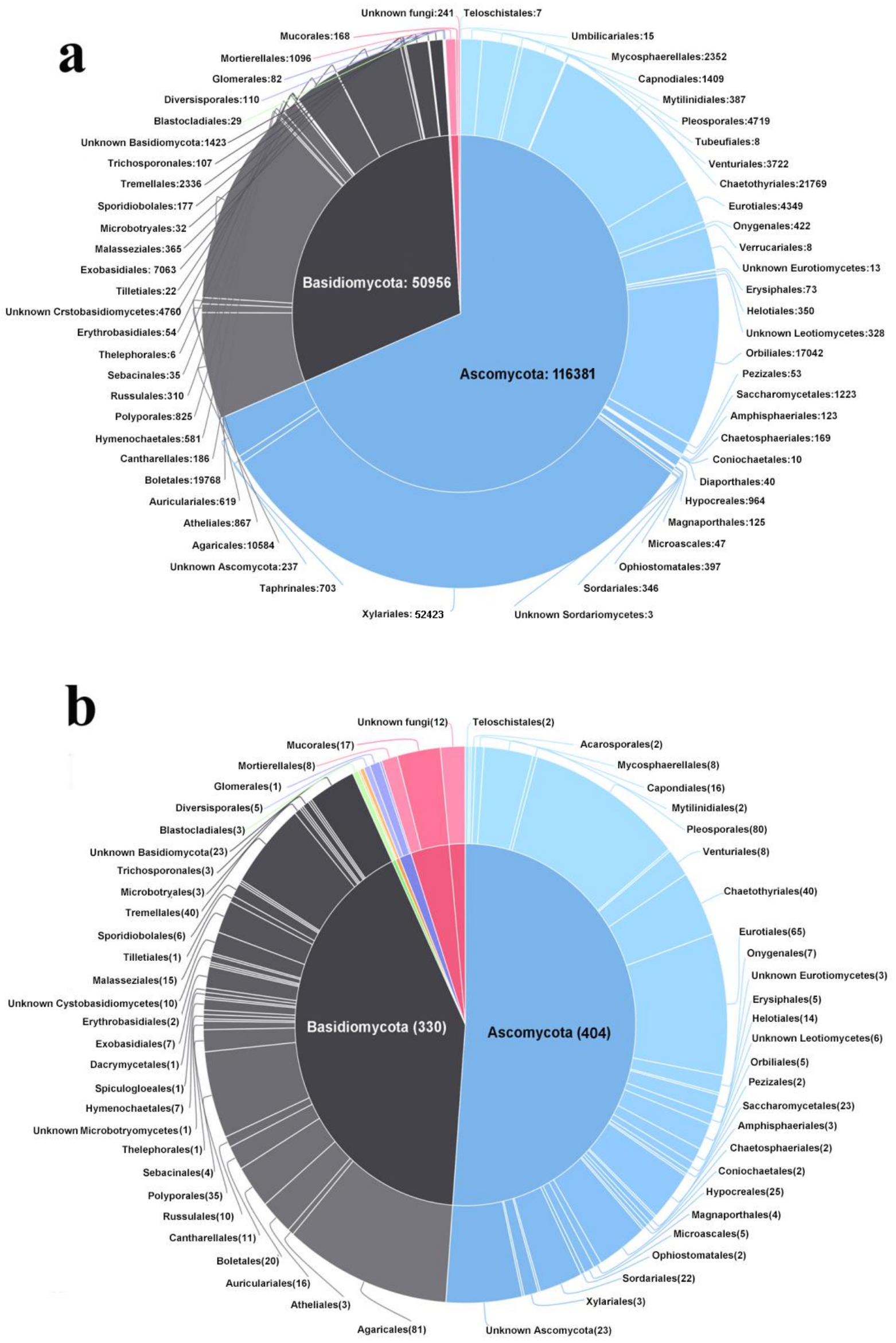

Fig. 1 - Pie charts showing the taxonomic distribution of sequences (reads) and OTUs at the order level. a Taxonomic distribution of 169,149 reads. b Taxonomic distribution of 791 OTUs 

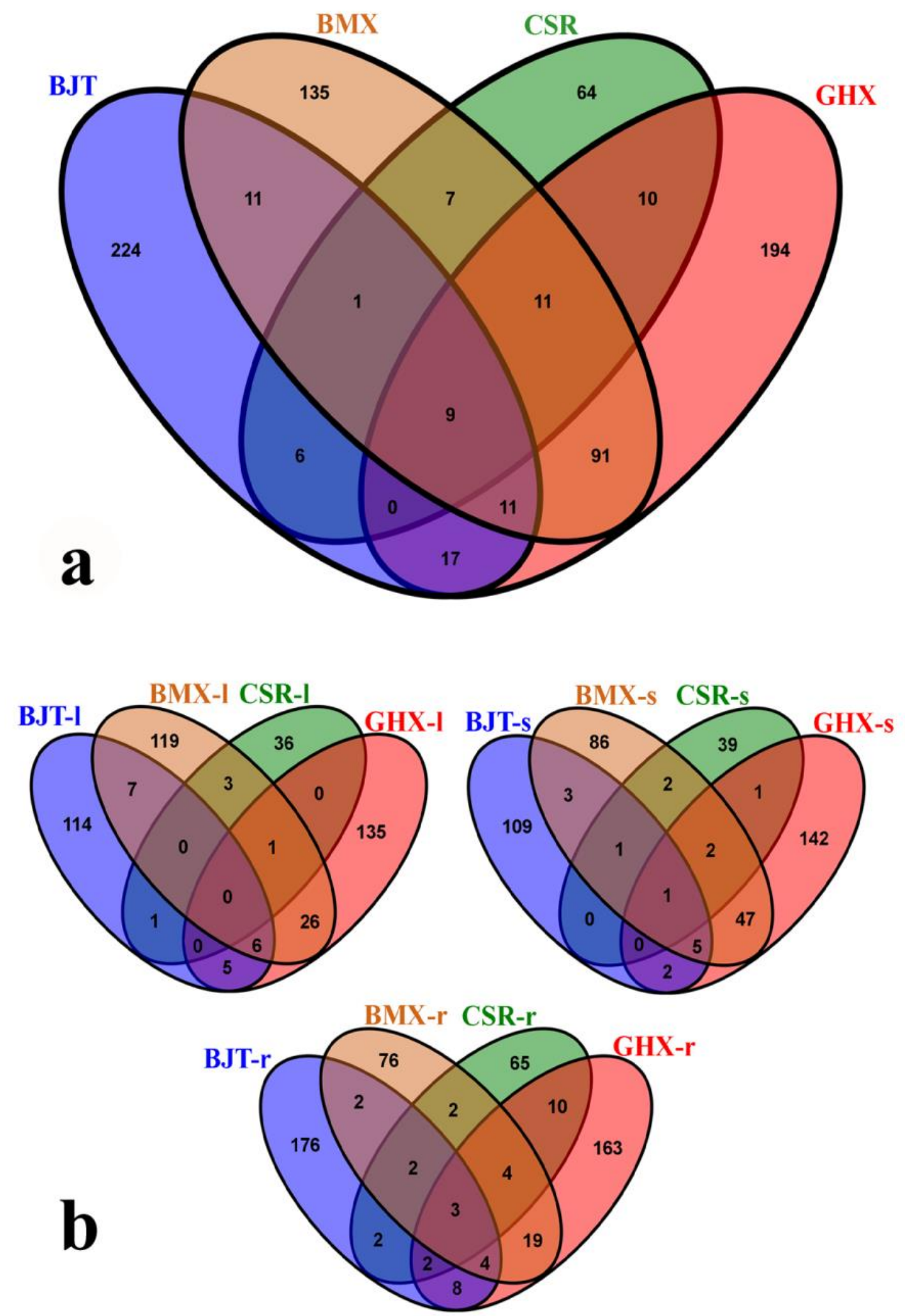

Fig. 2 - The differences and similarities of endophytes in four different plant species of tradition Chinese medicine (TCM). Numbers within the Venn diagrams represent the distinct OTUs number (non-overlap) and shared OTUs number (overlap). a Venn diagrams of endophytes in four different plant species. b Venn diagrams of endophytes in same organ among four different plants. BJT stands for Morinda officinalis, BMX stands for Aquilaria sinensis, CSR stands for Amomum villosum, GHX stands for Pogostemon cablin, and "-r" means roots of plant, "-s" means stems of plant, "-l" means leaves of plant. 


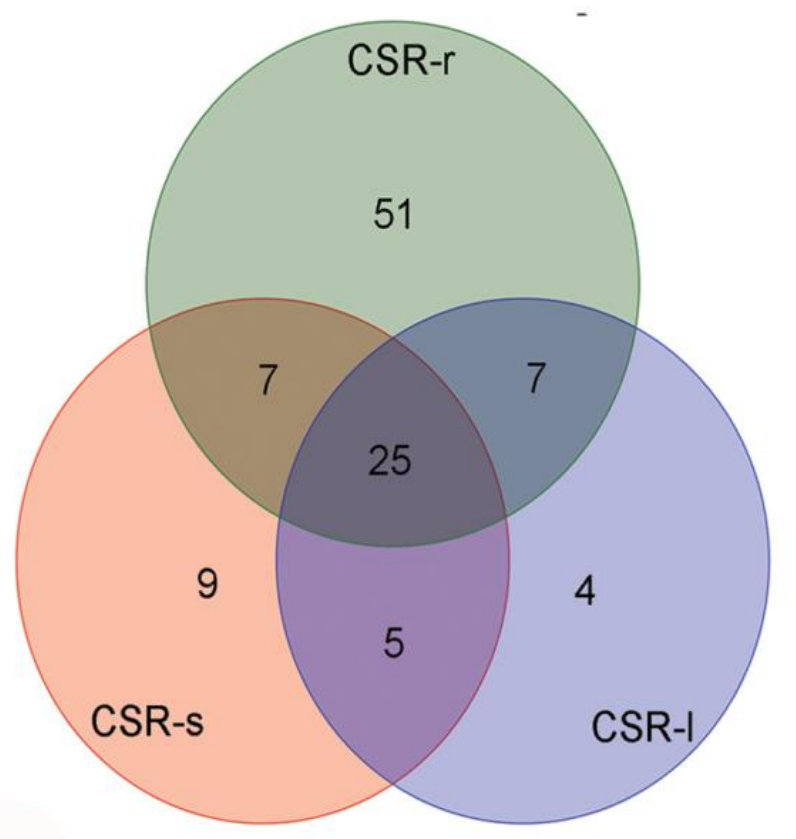

$\mathbf{a}$

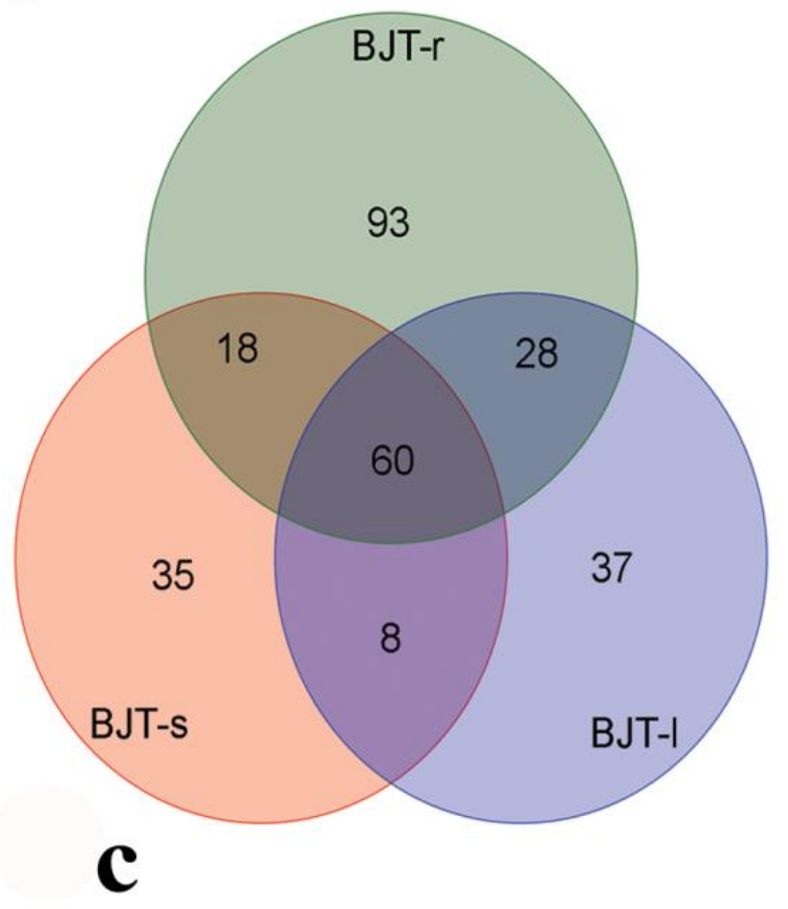

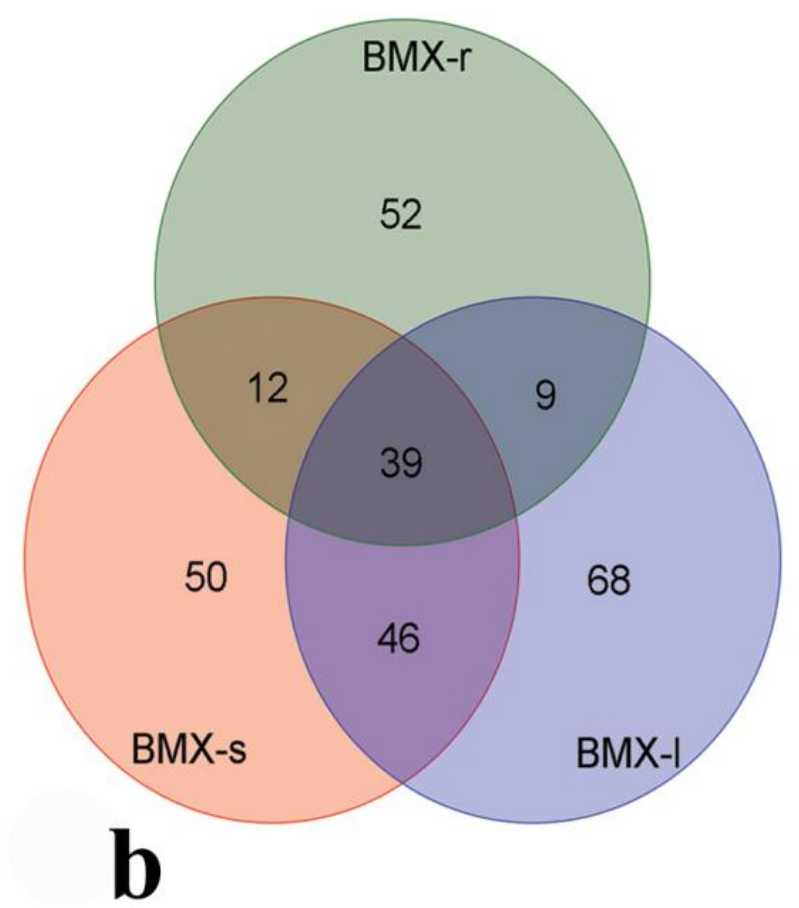

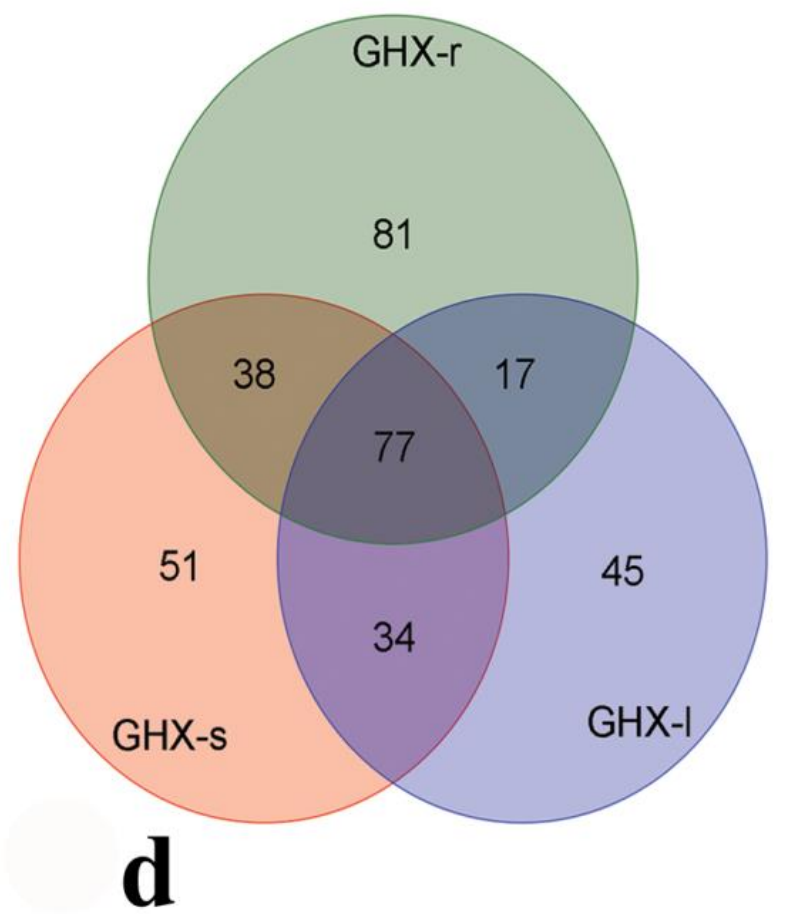

Fig. 3 - The differences and similarities of endophytes in different organs of the same plant species. Numbers within the Venn diagrams represent the distinct OTUs number (non-overlap) and shared OTUs number (overlap). a Venn diagrams of endophytes in Amomum villosum (CSR). b Venn diagrams of endophytes in Aquilaria sinensis (BMX). c Venn diagrams of endophytes in Morinda officinalis (BJT). d Venn diagrams of endophytes in Pogostemon cablin (GHX). "-r" means roots of plant, "-s" means stems of plant, "-1" means leaves of plant. 


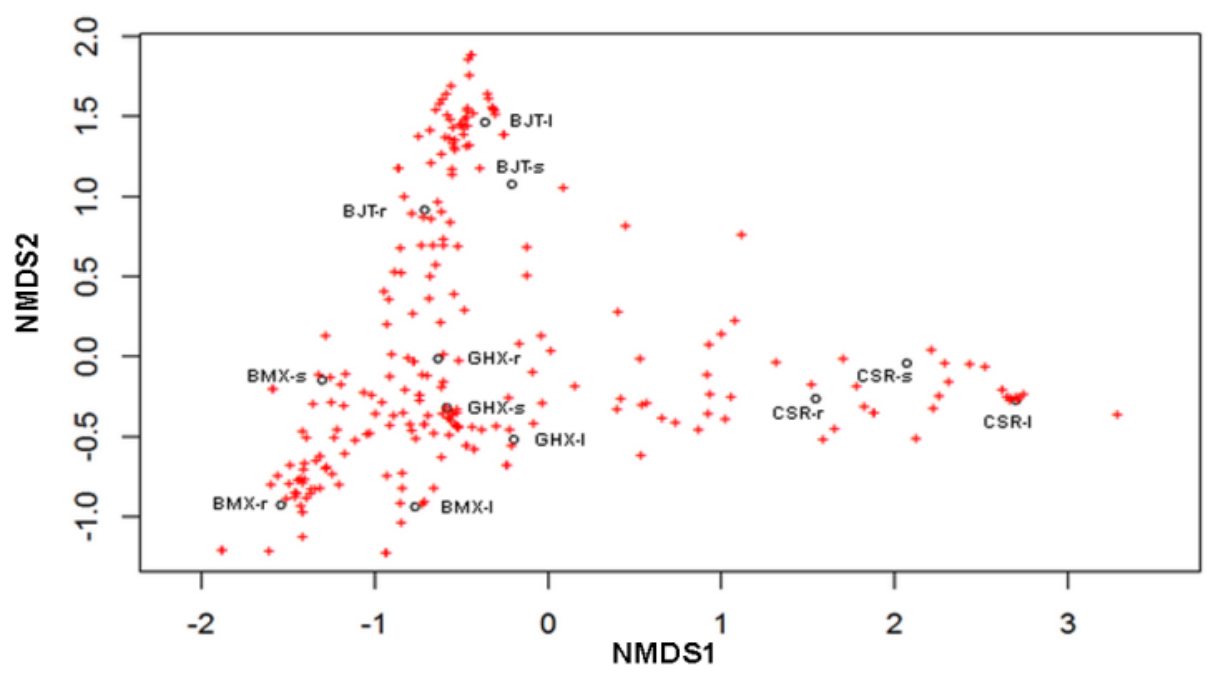

Fig. 4 - Nonmetric multidimensional scaling (NMDS) ordination based on Bray-Curtistransformed abundance data for OTUs. Each red cross corresponds to an OTU and each cycle corresponds to a sample. BJT stands for Morinda officinalis, BMX stands for Aquilaria sinensis, CSR stands for Amomum villosum, GHX stands for Pogostemon cablin, and "-r" means roots of plant, "-s" means stems of plant, "-l" means leaves of plant.

\section{Supplementary material}

Table S Overview of the 791 OTUs and their number of sequences found in the four medicinal plants

\section{Acknowledgements}

We thank Mr. Ming-zhi Li (Genepioneer Biotech Co., Ltd., Nanjing, China) for submitting the sequences; and Mrs. Jun-Ting Xie (Guangzhou Sagene Biotech Corp. Guangzhou, China) for technical assistance with statistics and bioesthetics. This work was supported by the National 973 Preliminary Project (No. 2014CB460613), the Field Scientific Experimental Station Project of Guangdong academy of science (No. Sytz201512), the Science and Technology Key Program of Guangzhou, China (201607020017), the Science and Technology Planning Project of Guangdong Province (Nos. 2014A030304050 and 2015A030302060).

\section{References}

Badali H, Gueidan C, Najafzadeh MJ, Bonifaz A et al. 2008 - Biodiversity of the genus Cladophialophora. Studies in Mycology 61, 175-191. https://doi.org/10.3114/sim. 2008. 61.18

Chen D, Song YL, Nie CX, Ma X, Tu PF. 2012 - Chemical constituents from Aquilaria sinensis (Lour.) Gilg. Journal of Chinese Pharmaceutical Sciences 21, 88-92.

Chen J, Zhang LC, Xing YM, Wang YQ et al. 2013 - Diversity and taxonomy of endophytic Xylariaceous fungi from medicinal plants of Dendrobium (Orchidaceae). Plos One 8(3), e58268. http://dx.doi.org/10.1371/journal.pone. 0058268

Chen ZB, Li B, Wang DK, Yu L et al. 2016 - Diversity and structure of endophytic bacteria in Menthae Haplocalycis Herba. Acta Agriculturae Zhejiangensis 28, 56-63.

Dreyfuss MM, Chapela IH. 1994 - Potential of fungi in the discovery of novel, low-molecular weight pharmaceuticals. In: Gullao VP, (ed) The discovery of natural products with therapeutic potential. Butterworth-Heinemann, London, pp. 49-80.

Duong LM, Jeewon R, Lumyong S, Hyde KD. 2006 - DGGE coupled with ribosomal DNA gene phylogenies reveal uncharacterized fungal phylotypes. Fungal Diversity 23, 121-138. 
Fu C, Chen C, Zhou GX, Ye WC. 2011 - Chemical constituents from fruits of Amomum villosum. Chinese Traditional and Herbal Drugs 42, 2410-2412.

Gardes M, Bruns TD. 1993 - ITS primers with enhanced specificity for basidiomycetes application to the identification of mycorrhizae and rusts. Molecular Ecology 2, 113-118. http://dx.doi.org/10.1111/j.1365-294X.1993. tb00005.x

Gong LJ, Guo SX. 2009 - Endophytic fungi from Dracaena cambodiana and Aquilaria sinensis and their antimicrobial activity. African Journal of Biotechnology 8, 731-736.

Guo LD, Hyde KD, Liew ECY. 2000 - Identification of endophytic fungi from Livistona chinensis based on morphology and rDNA sequences. New Phytologist 147, 617-630.

Higgins KL, Arnold AE, Miadlikowska J, Sarvate SD, Lutzoni F. 2007 - Phylogenetic relationships, host affinity, and geographic structure of boreal and arctic endophytes from three major plant lineages. Molecular Phylogenetics and Evolution 42, 543-555. http://dx.doi.org/10.1016/ j.ympev.2006.07.012

Li CG, Wu YG, Lin YF, Zhang JF et al. 2011 - Research progress on the chemical compounds of Pogostemon cablin (Blanco.) Benth. Jiangsu Agricultural Science 39, 498-500.

Li DL, Chen YC, Tao MH, Li HH, Zhang WM. 2012 - Two new octahydronaphthalene derivatives from Trichoderma spirale, an endophytic fungus derived from Aquilaria sinensis. Helvetica Chimica Acta 95, 805-809.

Lin MZ, Zheng S, Tian HQ. 2010 - Study situation and prospects of the research on Morinda officinalis. Subtropical Plant Science 39, 74-78.

Mohamed R, Jong PL, Zali MS. 2010 - Fungal diversity in wounded stems of Aquilaria malaccensis. Fungal Diversity 43, 67-74. http://dx.doi.org/10.1007/s13225-010- 0039-z

Mucciarelli M, Scannerini S, Bertea C, Maffei M. 2003 - In vitro and in vivo peppermint (Mentha piperita) growth promotion by nonmycorrhizal fungal colonization. New Phytologist 158, 579-591. http://dx.doi.org/10.1046/j.1469-8137.2003.00762.x

Nalini MS, Sunayana N, Prakash HS. 2014 - Endophytic fungal diversity in medicinal plants of Western Ghats, India. International Journal of Biodiversity 11, 1-9. http://dx.doi.org/ $10.1155 / 2014 / 494213$

Ning Y, Li YL, Zhou GY, Yang LC, Xu WH. 2016 - Community composition and diversity of endophytic fungi from roots of Sinopodophyllum hexandrum in forest of Upper-north mountain of Qinghai province. China Journal of Chinese Materia Medica 41, 1227-1234.

Ou M. 1989 - Chinese-English manual of common-used prescriptions in traditional Chinese medicine. Hongkong: Joint Publishing Company, pp. 1-89.

Ownley BH, Gwinn KD, Vega FE. 2010 - Endophytic fungal entomopathogens with activity against plant pathogens: ecology and evolution. Biocontrol 55, 113-128. http://dx.doi.org/ 10.1007/s10526-009-9241-x

Panda SK, Mohanta YK, Padhi L, Park YH et al. 2016 - Large scale screening of ethnomedicinal plants for identification of potential antibacterial compounds. Molecules 21, 293. http://dx.doi.org/10.3390/molecules21030293

Premalatha K, Kalra A. 2013 - Molecular phylogenetic identification of endophytic fungi isolated from resinous and healthy wood of Aquilaria malaccensis, a red listed and highly exploited medicinal tree. Fungal Ecology 6, 205-211. http://dx.doi.org/10.1016/j.funeco. 2013.01.005

Shekhawat KK, Rao DV, Batra A. 2013 - In vitro antimicrobial activities of endophytic fungi isolates from medicinal tree Melia azedarach L. Journal of Microbiology Research 3, $19-24$

Spadaro DC, Garibaldi A, Gullino ML. 2004 - First results on the use of molecular markers to track Metschnikowia pulcherrima strains against postharvest rots on golden delicious apple. The Plant Pathology Journal 15, 159-163.

Sun JQ, Guo LD, Zang W, Ping WX, Chi DF. 2008 - Diversity and ecological distribution of endophytic fungi associated with medicinal plants. Science in China Series C: Life Sciences 51, 751-759. http://dx.doi.org/10.1007/s11427-008-0091-z 
Sun X, Ding Q, Hyde KD, Guo LD. 2012 - Community structure and preference of endophytic fungi of three woody plants in a mixed forest. Fungal Ecology 5, 624-632. http://dx.doi.org/10.1016/j.funeco.2012.04.001

U'Ren JM, Lutzoni F, Miadlikowska J, Laetsch AD, Arnold AE. 2012 - Host and geographic structure of endophytic and endolichenic fungi at a continental scale. American Journal of Botany 99, 898-914.

Wang L, Zhang WM, Pan QL, Li HH et al. 2009 - Isolation and molecular identification of endophytic fungi from Aquilaria sinensis. Journal of Fungal Research 7, 37-42.

Wang M, Chen YC, Sun ZH, Tan GH et al. 2016 - Study on cytotoxic secondary metabolites of endophytic fungus Diaporthe longicolla A616 from Pogostemon cablin. China Journal of Chinese Materia Medica 41, 2112-2117.

Wang Y, Guo LD, Hyde KD. 2005 - Taxonomic placement of sterile morphotypes of endophytic fungi from Pinus tabulaeformis (Pinaceae) in northeast China based on rDNA sequences. Fungal Diversity 19, 59-64.

White TJ, Bruns T, Lee S, Taylor J. 1990 - Amplification and direct sequencing of fungal ribosomal RNA genes for phylogenetics. In: Innis MA, Gelfand DH, Sninsky JJ, White TJ (eds), PCR Protocols: a guide to methods and applications. Academic Press, New York, pp. 315-322.

Wu HY, Liu WQ, Zheng J, Li CM. 2016 - Summary of the climate of Guangdong province in 2015. Guangdong Meteorology 38, 1-5.

Zhang LG, Wei XY, Ma CX. 2011 - Endophytic fungi living in medicinal plants as new sources of bioactive substances. Pharmaceutical Biotechnology 18, 453-456.

Zhang T, Yao YF. 2015 - Endophytic fungal communities associated with vascular plants in the high arctic zone are highly diverse and host-plant specific. Plos One 10(6), e0130051. http://dx.doi.org/10.1371/journal.pone.0130051

Zhang YX. 2008 - Identification and diversity analysis of endophytic fungi in Pogostemon cablin and Andrographis paniculata. PhD Thesis, South China Agricultural University, China.

Zhang YX, Bei YS, You CP, Xiang MM. 2012 - Study on biodiversity of endophytic fungi of Morinda officinalis. Guangdong Agricultural Sciences 39, 23-25.

Zhang YX, Yang J, Huang JH, Xiang MM. 2010 - Study on biodiversity of endophytic fungi of Amomum villosum. Journal of Zhongkai University of Agriculture and Engineering 23, 15-17. 